\title{
Water contents of glasshouse soils at field capacity and at saturation. 1. Relationships between water contents
}

J. van den Ende

Glasshouse Crops Research Station, P.O. Box 8, NL 2670 AA Naaldwijk, Netherlands

Received 18 January 1988; accepted 21 March 1988

Key words: water contents, glasshouse soils, field capacity, pressure head, saturated soil, soil drying

\begin{abstract}
For glasshouse soils, the relationship between water contents at saturation and at field capacity was determined. To that end, soil samples were taken from 75 glasshouses located in the western part of the Netherlands. Sandy, loamy and peaty soils were about equally represented.

Water contents of soils at sampling time were found to correspond closely with those at field capacity. Water contents of saturated pastes obtained from fieldmoist soil samples were higher than those of saturated pastes obtained from soil samples dried previously. For the relationships between water contents of fieldmoist soil samples and of saturated pastes obtained from field-moist and dried soil samples, correlation coefficients of 0.986 and 0.985 , respectively, were found.
\end{abstract}

\section{Introduction}

For the determination of soil salinity, frequently use is made of a saturation extract. This extract is obtained through vacuum filtration of a water-saturated soil paste.

Richards (1954) recommended the use of saturation extracts for the purpose mentioned, as he considered the water content at saturation to be directly related to that in the field. In this view, the water content at saturation would be approximately twice that at field capacity, and, consequently, the salt concentration would tend to be about half. Subsequently, however, several workers (Geraldson, 1957; Longenecker \& Lyerly, 1964; Arnold Bik, 1970; Bower et al., 1975) found other water content ratios. If, therefore, the saturation extract is to be used, it is important to determine first how the water content at saturation is related to that in the field.

In order to check whether the saturation extract could present information on the chemical composition of soil solution, the relationship between water contents at saturation and at field capacity was investigated for glasshouse soils at the Glass- 
house Crops Research Station. In this investigation, it was further examined whether the water content at saturation is affected by drying the soil prior to saturating it. As far as known, researchers have not yet paid attention to such an influence, despite the fact that such drying is often practised.

In the investigation, the water contents of field-moist soil and of saturated pastes prepared from field-moist soil and from dried soil were determined. As the soils sampled possessed adequate internal drainage and had been frequently and amply watered prior to sampling, it was assumed that the water contents of the soils at sampling time were approximately equal to those at field capacity. The validity of this assumption was checked by determining the water contents of undisturbed soil samples at a pressure head of $-63 \mathrm{~cm}(\mathrm{pF} 1.8)$. This pressure head value was deduced from the depth at which the tile drains in the soils were situated.

In this first part of the paper, various relationships between water contents found are discussed.

\section{Materials and methods}

\section{Selection of the soils to be examined}

Soil samples were taken from 75 different glasshouses used for tomato growing. The glasshouses were located in the western part of the Netherlands. Pedologically speaking, the soils are young. The parent materials consist of mineral sediments of predominantly marine origin, of organic sediments and of peat formed in situ.

Based on the mass fraction organic matter of oven-dry soil $\left(f_{\mathrm{H}}\right)$, the soils were grouped as follows: $f_{\mathrm{H}}<0.1$ (soil type classes 1,2 and 3 ), $f_{\mathrm{H}}$ between 0.1 and 0.2 (class 4 ) and $f_{\mathrm{H}}>0.2$ (class 5 ). The first category was subdivided according to the mass fraction clay of oven-dry soil $\left(f_{C}\right)$ as follows: $f_{C}<0.05$ (class 1 ), $f_{C}$ between 0.05 and 0.15 (class 2 ) and $f_{C}>0.15$ (class 3 ). The numbers of soils in the soil type classes $1,2,3,4$ and 5 were $12,14,17,19$ and 13 , respectively.

In Table 1, mean values and ranges of some physical and chemical characteristics of the soils of the five soil type classes are listed.

\section{Methods of soil sampling and of handling samples}

The water contents of the soils to be sampled were estimated by feeling. Generally, the soils were found to be close to field capacity as a result of the fact that these glasshouse soils were frequently and amply watered and possessed adequate internal drainage. Ordinarily, watering took place with the aid of sprinkling lines. In some soils, artificial drainage was absent because of excellent natural drainage, but most soils were equipped with a tile drainage system. The tiles were present at a depth of approximately $80 \mathrm{~cm}$, and the distance between the lines was usually 6.4 $\mathrm{m}$, and sometimes $3.2 \mathrm{~m}$.

When soils were judged to be at field capacity, they were sampled immediately. Sometimes, however, soils were considered to be somewhat below field capacity. In these cases, sampling was postponed until the next watering had taken place. 


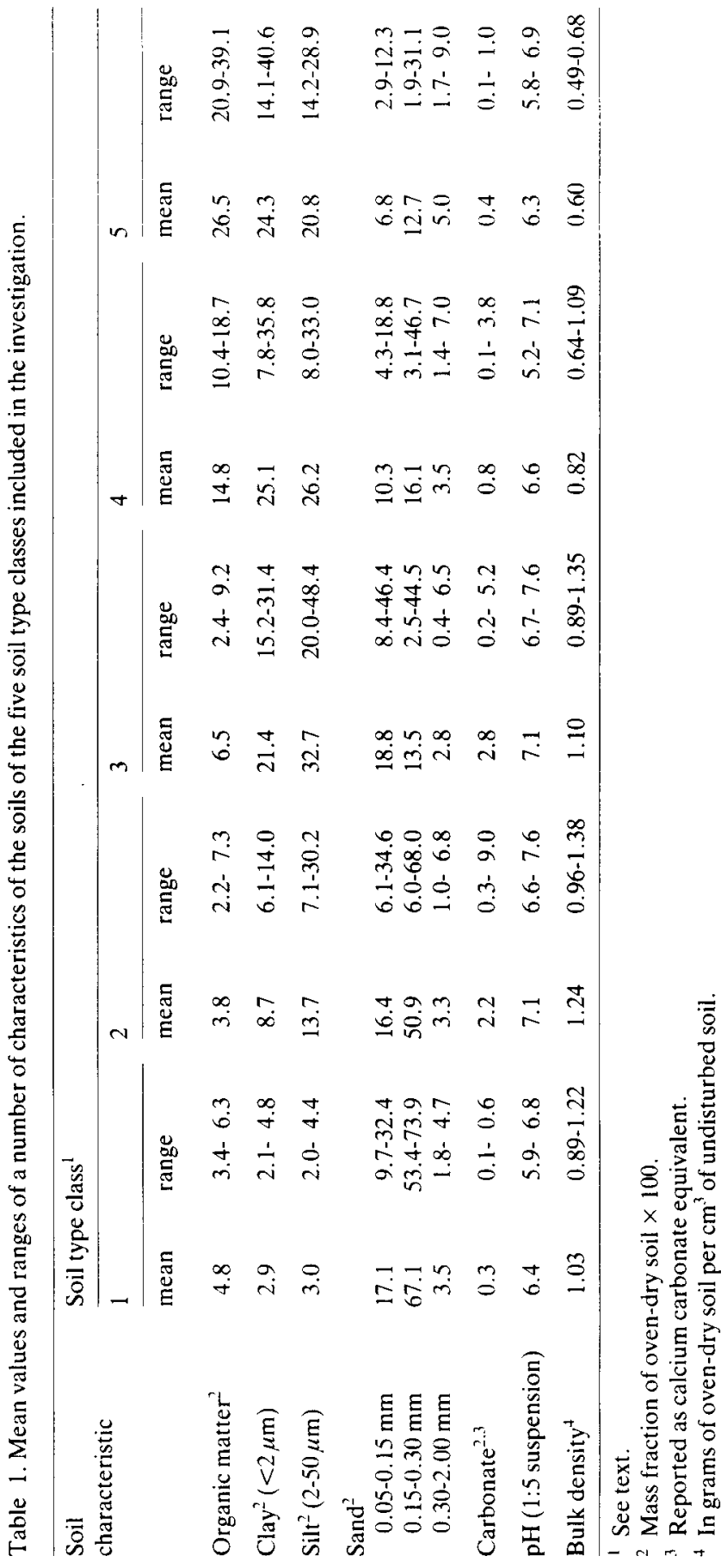


Per sampling site, one sample of disturbed soil and three samples of undisturbed soil were taken. The undisturbed samples were used for determining water content at a pressure head of $-63 \mathrm{~cm}$ and bulk density (mass of oven-dry soil per volume of undisturbed soil).

The disturbed samples were taken from the $5-30 \mathrm{~cm}$ layer, excluding the upper 5 $\mathrm{cm}$ layer because of frequent deviations in properties. The undisturbed samples were taken with the aid of sampling cores. The 5 -cm diameter cores had a content of $100 \mathrm{~cm}^{3}$. All core samples were collected from the $15-20 \mathrm{~cm}$ layer.

In the laboratory, the disturbed samples were split into two subsamples. One subsample was kept in field-moist condition and the other was dried overnight in a drying cabinet at $50{ }^{\circ} \mathrm{C}$. Subsequently, the dried soil was ground and stored. The soil thus treated will be called air-dry soil.

\section{Preparation of saturated soil pastes}

Saturated soil pastes were obtained from both field-moist and air-dry soil samples, according to Richards (1954). After storing the pastes overnight at $25^{\circ} \mathrm{C}$, the criteria of saturation were rechecked. A little water had to be added to many pastes to restore the condition of saturation. For the pastes derived from peaty soils, such an addition was practically always necessary. The volume of additional water needed was larger for pastes prepared from air-dry soil than for those prepared from fieldmoist soil. The need to add more water was also present for pastes prepared from air-dry loamy soils, in contrast to pastes prepared from field-moist loamy soils. Pastes from sandy soils did not require any additional water. On the contrary, with many of these pastes, some supernatant water accumulated overnight. However, with the stirring employed in rechecking the criteria of saturation this free water disappeared.

\section{Determination of physical and chemical characteristics}

The following characteristics of the soils used in the investigation were determined.

Mass fraction organic matter of oven-dry soil. The method employed to determine organic matter was based on a determination of organic carbon according to a drycombustion procedure. Organic-carbon content was converted to organic-matter content by using the conventional convursion factor 1.724 (Broadbent, 1953).

Mass fractions clay, silt and sand of oven-dry soil. Particles with effective diameter exceeding $16 \mu \mathrm{m}$ were partitioned by dry sieving into a large number of fractions, after which the weights of the various fractions were determined. The fractions of particles smaller than $2 \mu \mathrm{m}$ and between 2 and $16 \mu \mathrm{m}$ were determined with the pipette method.

Mass fraction carbonate of oven-dry soil. Carbonate was determined with the volumetric calcimeter method. Results are reported as calcium carbonate equivalent. 
$p H . \mathrm{pH}$ was measured in a 1:5 aqueous suspension (by weight) after storage overnight.

Mass ratio 'water/solid phase' of field-moist and saturated soil. The mass ratios 'water/solid phase' of field-moist and saturated soil samples were determined by drying overnight at $105^{\circ} \mathrm{C}$.

Mass ratio 'water/solid phase' at a pressure head of $-63 \mathrm{~cm}$ and bulk density. The core samples were saturated with water and, next, adjusted to a pressure head of $-63 \mathrm{~cm}$ with the aid of the sand-box method. The mass ratios 'water/solid phase' and the bulk density values were determined by drying the adjusted core samples during 24 h at $105^{\circ} \mathrm{C}$.

Mass fractions organic matter, clay, silt, sand and carbonate were determined by standard methods in the Soils Laboratory of the IJsselmeerpolders Development Authority, Lelystad, Netherlands, the other characteristics in our own soils laboratory.

Most determinations were carried out on air-dry soil samples. However, fieldmoist soil samples were used for determining bulk density, mass ratio 'water/solid phase' at a pressure head of $-63 \mathrm{~cm}$ and mass ratio 'water/solid phase' of field-moist soil and of saturated soil prepared from field-moist soil.

Determinations were made in duplicate, except those for which small quantities of field-moist soil were used, being the determinations of mass ratio 'water/solid phase' of field-moist soil, mass ratio 'water/solid phase' at a pressure head of -63 $\mathrm{cm}$, and bulk density. These were carried out in triplicate.

Symbols

For water contents expressed as mass ratios 'water/solid phase', the symbol $w$ will be used. The subscripts $\mathrm{f}$, sf, sa and -63 stand for field-moist soil, saturated soil obtained from field-moist soil, saturated soil obtained from air-dry soil and soil at a pressure head of $-63 \mathrm{~cm}$, respectively.

\section{Results and discussion}

Water content of field-moist soil and water content at a pressure head of $-63 \mathrm{~cm}$

A summary of the $w_{\mathrm{f}}$ and $w_{-63}$ values found is given in Table 2 . The $w_{\mathrm{f}}$ values were approximately equal to the $w_{-63}$ values.

In the soils, the depths of the water tables probably did not differ much from those of the tile drains (approximately $80 \mathrm{~cm}$ ). For each of the soils, therefore, it may be expected that at field capacity the pressure head at each elevation above the water table was about equal to the negative value of the distance to the water table. In this way, it is found that at field capacity the average pressure head value of the $5-30 \mathrm{~cm}$ soil layer was about $-62.5 \mathrm{~cm}$ (the average depths of the drain tiles and of 
Table 2. Mean and extreme values of the water contents of field-moist samples $\left(w_{f}\right)$ and undisturbed samples at a pressure head of $-63 \mathrm{~cm}\left(w_{-6.3}\right)$ of soils in five soil type classes.

\begin{tabular}{|c|c|c|c|c|}
\hline \multirow{2}{*}{$\begin{array}{l}\text { Soil } \\
\text { type } \\
\text { class }^{2}\end{array}$} & \multicolumn{2}{|l|}{$w_{\mathrm{f}}^{1}$} & \multicolumn{2}{|l|}{$w_{-63}{ }^{1}$} \\
\hline & mean & range & mean & range \\
\hline 1 & 0.276 & $0.204-0.345$ & 0.272 & $0.195-0.349$ \\
\hline 2 & 0.237 & $0.177-0.328$ & 0.241 & $0.178-0.332$ \\
\hline 3 & 0.368 & $0.240-0.469$ & 0.368 & $0.231-0.506$ \\
\hline 4 & 0.585 & $0.431-0.796$ & 0.576 & $0.419-0.751$ \\
\hline 5 & 0.848 & $0.715-1.111$ & 0.809 & $0.720-1.019$ \\
\hline
\end{tabular}

'Mass ratio 'water/solid phase'.

${ }^{2}$ See text.

the $5-30 \mathrm{~cm}$ layer being circa $80 \mathrm{~cm}$ and $17.5 \mathrm{~cm}$, respectively). Therefore, it may be concluded that at sampling time the water contents of the soils corresponded closely to those at field capacity. This conclusion is only valid if some assumptions made are substantially correct. First, it was assumed that the relationship between pressure head and water content of a soil in situ is principally characterized by the so-called drying curve. Further, it was assumed that the circumstances prevailing in the core samples during the adjustment to a pressure head of $-63 \mathrm{~cm}$ did not differ essentially from those in the soils in situ. It was supposed, for instance, that differences in temperature between the two circumstances were too small to affect the relationship between pressure head and water content materially and also that for the soils in situ this relationship was hardly influenced by an overburden pressure.

The largest differences between $w_{\mathrm{f}}$ and $w_{-63}$ values occurred in soil type class 5 . On average, the water tables of the soils in this class were maintained at a somewhat smaller depth than in the other classes. Accordingly, in soil type class 5 the $w_{\mathrm{f}}$ values were slightly higher than the $w_{-63}$ values.

The following regression equation was found for the relationship between $w_{\mathrm{f}}$ and $w_{-63}$ values:

$$
w_{\mathrm{f}}=1.047 w_{-63}-0.012
$$$$
r=0.987
$$

The deviation of the regression coefficient from 1 and the deviation of the intercept from 0 can be ascribed to the relatively high $w_{\mathrm{f}}$ values of soils in class 5 .

\section{Water contents of saturated soils obtained from field-moist soil and from air-dry soil}

The $w_{\mathrm{sf}}$ and $w_{\mathrm{sa}}$ values found and their ratios are summarized in Table 3. The $w_{\mathrm{sa}}$ values were lower than the $w_{\mathrm{sf}}$ values. The $w_{\mathrm{sa}} / w_{\mathrm{sf}}$ quotients averaged 0.85 . Thus, it appeared to make a difference whether field-moist or air-dry soil samples were used for preparing saturated soil pastes. Method 2 of the US Salinity Laboratory (Richards, 1954) is at variance with this finding. According to the description of this method, both field-moist and air-dry soil samples can be used for preparing satu- 
Table 3. Mean and extreme values of the water contents of saturated soil pastes obtained by saturating field-moist $\left(w_{\mathrm{sf}}\right)$ and air-dry $\left(w_{\mathrm{sa}}\right)$ samples of soils in five soil type classes and mean and extreme values of the $w_{\text {sa }} / w_{\text {sf }}$ quotients.

\begin{tabular}{|c|c|c|c|c|c|c|}
\hline \multirow{2}{*}{$\begin{array}{l}\text { Soil } \\
\text { type } \\
\text { class }^{2}\end{array}$} & \multicolumn{2}{|l|}{$w_{\mathrm{sf}}^{1}$} & \multicolumn{2}{|l|}{$w_{\mathrm{sa}}{ }^{1}$} & \multicolumn{2}{|c|}{$w_{\mathrm{sa}} / w_{\mathrm{sf}}$} \\
\hline & mean & range & mean & range & mean & range \\
\hline 1 & 0.500 & $0.420-0.594$ & 0.437 & $0.380-0.498$ & 0.88 & $0.83-0.95$ \\
\hline 2 & 0.411 & $0.328-0.561$ & 0.359 & $0.293-0.462$ & 0.88 & $0.78-0.97$ \\
\hline 3 & 0.674 & $0.430-0.890$ & 0.566 & $0.381-0.743$ & 0.84 & $0.79-0.89$ \\
\hline 4 & 0.944 & $0.691-1.154$ & 0.786 & $0.591-0.944$ & 0.84 & $0.80-0.89$ \\
\hline 5 & 1.249 & $1.047-1.627$ & 1.014 & $0.854-1.312$ & 0.81 & $0.73-0.90$ \\
\hline
\end{tabular}

${ }^{1}$ Mass ratio 'water/solid phase'.

2 See text.

ration extracts. However, the description does not mention that the water content at saturation may be different in these two cases.

The relationship between $w_{\mathrm{sf}}$ and $w_{\mathrm{sa}}$ values can be described well by a linear function. The following regression equation was calculated:

$$
w_{\mathrm{sa}}=0.772 w_{\mathrm{sf}}+0.049
$$$$
r=0.994
$$

In the second part of this paper (van den Ende, 1988), an explanation for the difference found between $w_{\mathrm{sf}}$ and $w_{\mathrm{sa}}$ will be given.

\section{Relationship between water contents of saturated soil and of field-moist soil}

A summary of the $w_{\mathrm{sP}} / w_{\mathrm{f}}$ and $w_{\mathrm{sa}} / w_{\mathrm{f}}$ quotients is given in Table 4 . These quotients varied among soil type classes. On average, the soils in classes 4 and 5 had lower quotients than those in other classes. The quotient values varied also within one class.

It is often assumed that for most soils the water content at saturation is approxi-

Table 4. Mean and extreme values of the quotients of the water contents of saturated and field-moist samples of soils in five soil type classes.

\begin{tabular}{|c|c|c|c|c|}
\hline \multirow{2}{*}{$\begin{array}{l}\text { Soil } \\
\text { type } \\
\text { class }^{2}\end{array}$} & \multicolumn{2}{|c|}{$w_{\mathrm{sf}} / w_{\mathrm{f}}^{\prime}$} & \multicolumn{2}{|c|}{$w_{\mathrm{sa}} / w_{\mathrm{f}}^{1}$} \\
\hline & mean & range & mean & range \\
\hline 1 & 1.83 & $1.45-2.13$ & 1.60 & $1.28-1.86$ \\
\hline 2 & 1.76 & $1.52-2.04$ & 1.54 & $1.26-1.84$ \\
\hline 3 & 1.82 & $1.60-2.06$ & 1.54 & $1.36-1.71$ \\
\hline 4 & 1.62 & $1.41-1.76$ & 1.35 & $1.17-1.49$ \\
\hline 5 & 1.47 & $1.39-1.62$ & 1.20 & $1.08-1.33$ \\
\hline
\end{tabular}

1 See for $w_{\mathrm{f}}, w_{\mathrm{sf}}$ and $w_{\mathrm{sa}}$ Tables 2 and 3 .

2 See text. 


\section{J. VAN DEN ENDE}

mately twice that at field capacity and four times that at the wilting point (Richards, 1954; Bernstein, 1962). As was shown above, the water contents of the soils at sampling time corresponded closely to those at field capacity, while the pressure head values of the topsoils amounted to approximately $-0.6 \mathrm{~m}$. Such a value is rather common for soils at field capacity (Franzmeier et al., 1960; Webster \& Beckett, 1972). From the quotients found the conclusion can, therefore, be drawn that a value of 2 is too high, certainly for soils rich in organic matter and for saturated pastes prepared from air-dry soil. Other workers (Geraldson, 1957; Bower et al., 1975) also found values lower than 2.

The saturation extract method has been criticized on account of the assertion that for many soils the water content at saturation is not simply related to that in the field (Longenecker \& Lyerly, 1964; Arnold Bik, 1970). The quotients found in the pres-

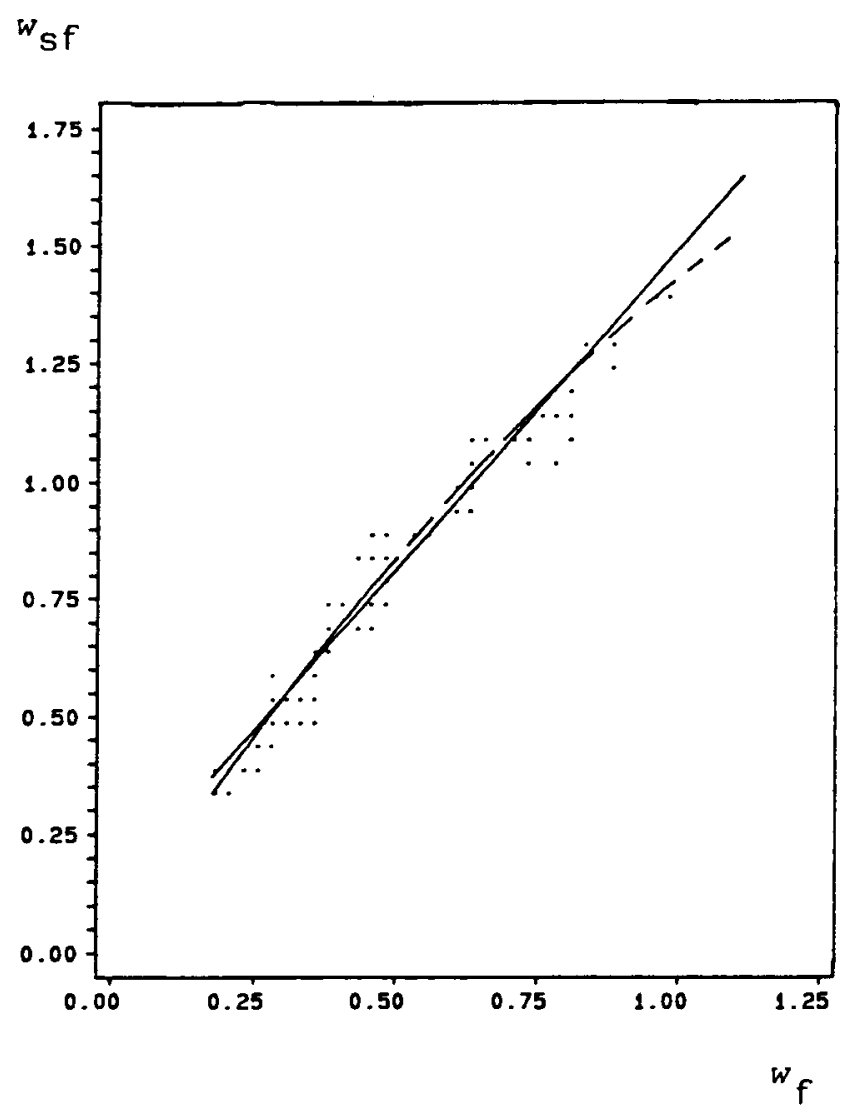

Fig. 1. Relationship between mass ratios 'water/solid phase' of field-moist soil samples $\left(w_{f}\right)$ and those of saturated soil pastes obtained from the field-moist samples $\left(w_{\text {sf }}\right)$. Functions adjusted:
linear (-)
$y=1.360 x+0.130$
$r=0.984$
curvilinear (-- ) $\quad y=-0.428 x^{2}+1.834 x+0.025$
$R=0.986$ 
ent experiment confirm that this criticism has a basis of truth. However, the differences among these quotients appear to remain within acceptable bounds, the more so as they can be partly accounted for by differences in organic-matter contents.

In view of the fact that $w_{\mathrm{sf}} / w_{\mathrm{f}}$ quotients depended somewhat on the levels of $w_{\mathrm{sf}}$ and $w_{\mathrm{f}}$ values, no completely linear relationship between the latter values may be expected. The same applies to $w_{\mathrm{sa}}$ and $w_{\mathrm{f}}$ values. Both the relationship between $w_{\mathrm{st}}$ and $w_{\mathrm{f}}$ values and that between $w_{\mathrm{sa}}$ and $w_{\mathrm{f}}$ values can be represented well by a quadratic function. It should be noted, however, that representation by a linear function is only a little less precise. The following regression equations were calculated:

$$
\begin{array}{lr}
w_{\mathrm{sf}}=1.360 w_{\mathrm{f}}+0.130 & r=0.984 \\
w_{\mathrm{sf}}=-0.428 w_{\mathrm{f}}^{2}+1.834 w_{\mathrm{f}}+0.025 & R=0.986 \\
w_{\mathrm{sa}}=1.053 w_{\mathrm{f}}+0.148 & r=0.981 \\
w_{\mathrm{sa}}=-0.444 w_{\mathrm{f}}^{2}+1.544 w_{\mathrm{f}}+0.039 & R=0.985
\end{array}
$$

The relationship between $w_{\mathrm{sf}}$ and $w_{\mathrm{f}}$ values is depicted in Fig. 1 .

\section{Conclusions}

Water contents of sampled topsoils in glasshouses used for tomato growing turned out to be approximately equal to those at a pressure head of $-63 \mathrm{~cm}(\mathrm{pF} 1.8)$. Water contents of saturated pastes obtained from field-moist soil were higher than those of saturated pastes obtained from previously dried soil. Water contents of the former pastes were on average 1.70 times higher than those of field-moist soil. For the latter pastes, the multiplication factor was 1.44 .

For the relationships between water contents of field-moist soil and those of saturated pastes obtained from field-moist and dried soil, correlation coefficients of 0.986 and 0.985 , respectively, were found. These high values show that the saturation extract can probably be used successfully as a means to determine the fertility status of a glasshouse soil.

The saturation extract is often used without any knowledge of the relationship between water contents at saturation and in field-moist condition. For optimum use of information on the fertility status of a soil analysed at saturation, it is necessary to know the relationship between the water content at saturation and that at field capacity. Before use is to be made of saturated pastes, it should be decided whether for the preparation of saturated soil pastes dried or field-moist soil samples should be employed.

\section{References}

Arnold Bik, R.. 1970. Nitrogen, salinity, substrates and growth of gloxinia and chrysanthemum. Agricultural Research Reports 739.89 pp. Pudoc, Wageningen.

Bernstein, L., 1962. Salt-affected soils and plants. Arid Zone Research 18: 139-174 
Bower, C. A., B. A. Kratky \& N. Ikeda, 1975. Growth of tomato on a tropical soil under plastic cover as influenced by irrigation practice and soil salinity. Journal of the American Society for Horticultural Science 100: 519-521.

Broadbent. F. E., 1953. The soil organic fraction. Advances in Agronomy 5: 153-183.

Ende, J. van den, 1988. Water contents of glasshouse soils at field capacity and at saturation. 2. Estimating water contents from organic-matter and clay contents or from loss-on-ignition. Netherlands Journal of Agricultural Science 36: 275-282.

Franzmeier, D. P., E. P. Whiteside \& A. E. Erickson, 1960. Relationship of texture classes of fine earth to readily available water. Transactions 7 th International Congress of Soil Science (Madison), Vol. 1, p. 354-363.

Geraldson, C. M., 1957. Soil soluble salts. Determination of and association with plant growth. Proceedings of the Florida State Horticultural Society 70: 121-126.

Longenecker, D. E. \& P. J. Lyerly, 1964. Making soil pastes for salinity analysis. A reproducible capillary procedure. Soil Science 97: 268-275.

Richards, L. A. (Ed.), 1954. Diagnosis and improvement of saline and alkali soils. Agriculture Handbook No 60. $160 \mathrm{pp}$. United States Department of Agriculture, US Government Printing Office, Washington, DC.

Webster. R. \& P. H. T. Beckett, 1972. Matric suctions to which soils in South Central England drain. Journal of Agricultural Science, Cambridge 78: 379-387. 